\title{
Development of a clinical prediction model of chronic kidney disease in primary Focal Segmental Glomerulosclerosis
}

Shahrzad Ossareh ( $\square$ ossareh_s@hotmail.com )

Iran University of Medical Sciences https://orcid.org/0000-0003-3020-9465

Mansoureh Yahyaei

Hasheminejad Kidney Center, Tehran

Mojgan Asgari

Hasheminejad Kidney Center

Hanri Afghahi

Skaraborg Hospital, Skövde

Research article

Keywords: Focal segmental glomerulosclerosis, End stage kidney disease, Pathology

Posted Date: January 21st, 2020

DOI: https://doi.org/10.21203/rs.2.11407/v2

License: (c) (i) This work is licensed under a Creative Commons Attribution 4.0 International License.

Read Full License 


\section{Abstract}

Background: Focal segmental glomerulosclerosis (FSGS) is one of the important causes of end stage kidney disease (ESKD). We evaluated the risk factors of progression of primary FSGS to chronic kidney disease (CKD) or ESKD with a predictive model including clinical and histological predictors. Methods: 201 patients with primary FSGS (59\% male, mean age: $38 \pm 15$ years), were studied. Time-dependent Cox model and $\mathrm{C}$ statistics were used for the predictive model. Interaction and correlation between independent variables were estimated. Results: During $55 \pm 27$ months of follow-up, 82 patients $(41 \%)$ developed CKD (46) or ESKD (36) patients. In adjusted model, 1 unit of higher serum creatinine (SCr) at baseline (HR:1.39, 95\% Cl: 1.15-1.70) and 1\% increase in glomeruli with segmental glomerulosclerosis (SGS) (HR: $1.03,95 \% \mathrm{Cl}: 1.02-1.04)$ or interstitial fibrosis/tubular atrophy (IF/TA) (HR: $1.03,95 \%$ Cl: 1.011.05) increased the risk of CKD/ESKD. In adjusted model, higher baseline proteinuria and collapsing variant were not associated with risk of CKD/ESKD. By adding SGS and IF/TA scores to baseline SCr in the model, discrimination by $\mathrm{C}$ statistics was $0.83(95 \% \mathrm{Cl}$ : $0.77-0.90)$ for prediction of CKD/ESKD . Median renal survival was 3.1 years ( $95 \% \mathrm{Cl}$ : 2.2-4.1 years) in patients with highest risks score (baseline eGFR<25 ml/min/1.73 m $2+$ IF/TA/SGS $>50 \%$ ), and 8.1 years (95\% Cl: 7.7-8.6 years).in those with lowest score (baseline eGFR $>75 \mathrm{ml} / \mathrm{min} / 1.73 \mathrm{~m} 2+\mathrm{IF} / \mathrm{TA} / \mathrm{SGS}<5 \%$ ). Conclusion: In primary FSGS, higher baseline SCr, increased SGS and IF/TA were the predictors for CKD/ESKD. Baseline proteinuria did not predict the risk of CKD/ESKD. Collapsing variant did not increase the risk of CKD/ESKD after adjustment for IF/TA score. These findings indicated the importance of baseline GFR and the degree of chronicity at biopsy as predictors of kidney outcome .

\section{Background}

Idiopathic or primary focal segmental glomerulosclerosis (FSGS) is a syndrome with proteinuria, mostly nephrotic range, focal and segmental glomerular sclerosis lesions and foot process effacement (1-4). (FSGS) is known as a one of the leading glomerular causes of end stage kidney disease (ESKD) in most parts of the world (5-7). In a recent report by our group the prevalence of FSGS rose to second rank among primary glomerular diseases (8). However in reports from many countries FSGS is the most common glomerulopathy diagnosed by kidney biopsy $(9,10)$. In patients with primary FSGS response to treatment or progression to chronic kidney disease (CKD) are very diverse and it has been reported that more than half of the patients progressed to end-stage kidney disease (ESKD) after 10 years of followup(7).

Columbia morphologic classification, which was defined in 2004, is the most popular method of classification of FSGS, and classifies FSGS to five pathologic variants including collapsing, cellular, tip lesion, perihilar and not otherwise specified (NOS)(1).It has been suggested that the histologic subtypes of FSGS correlate with remission and renal outcome $(11,12)$.

Identifying the risk factors that predict the progression of primary FSGS to CKD/ESKD could enable appropriate patient care and improved individualized decision-making. The aim of this study was to 
develop a model of prediction of chronic kidney disease (CKD)/ End- Stage Kidney Disease(ESKD) (dialysis or kidney transplantation) in patients with primary FSGS by baseline clinical, laboratory and pathological findings as predictors.

\section{Methods}

In this retrospective cohort study, the baseline clinical and laboratory data and the last proteinuria and serum creatinine of 356 patients with FSGS, who had been biopsied between 2005 and 2014 was extracted from the glomerulonephritis database of our hospital. The mean follow-up was $55 \pm 27$ months. The exclusion criteria were urinary reflux, single kidney, substance abuse, HIV disease, sickle cell anemia and other serious systemic diseases that would have caused secondary FSGS and age of less than 16 years old at the time of disease onset. Of 356 patients with diagnosis of FSGS, 78 patients were excluded from the study because of secondary disease, 77 were excluded because of information deficit and finally 201 patients were enrolled.

\section{Definitions:}

\section{Clinical definitions:}

Demographic data at admission: Demographic data at admission included age, sex, weight and clinical symptoms (hypertension and edema) and the result of laboratory parameters [serum creatinine (SCr), estimated GFR (eGFR), albumin, cholesterol and 24-hour urine protein]. The final SCr and 24 hour urine protein were recorded from outpatient charts and the outcome of treatment was defined according to the results of these parameters.

Complete remission: Final proteinuria $<300 \mathrm{mg} /$ day and $\mathrm{SCr}<1.4 \mathrm{mg} / \mathrm{dl}$.

Partial remission: Final proteinuria between 300 and to1999 mg/day and $<50 \%$ of baseline and SCr $<1.4$ $\mathrm{mg} / \mathrm{dl}$

No remission: Final proteinuria $\geq 2000 \mathrm{mg} /$ day and/or reduction of $<50 \%$ or SCr $\geq 1.4 \mathrm{mg} / \mathrm{dl}$.

Chronic kidney disease (CKD) at admission: $\mathrm{SCr} \geq 1.4 \mathrm{mg} / \mathrm{dl}$ for $\geq 3$ months before admission.

Progressive CKD, as the kidney outcome: $\mathrm{SCr} \geq 1.4 \mathrm{mg} / \mathrm{dl}$ and $\mathrm{SCr} \geq 50 \%$ of the baseline.

ESKD or death due to kidney disease, as the kidney outcome: ESKD was defined as the patient on maintenance dialysis or kidney transplant. Death due to kidney disease was defined as death on maintenance dialysis or kidney transplant due to the complications of either modality.

Arterial hypertension was defined as systolic BP $\geq 140 \mathrm{mmHg}$ and/or diastolic BP $\geq 90 \mathrm{mmHg}$.

Renal function expressed as estimated GFR (eGFR) ( milliliters/ minute/ $1.73 \mathrm{~m}^{2}$ ), was calculated by using the Modification of Diet in Renal Disease study equation (MDRD). Estimated GFR at baseline was 
categorized to four groups: $>75 \mathrm{ml} / \mathrm{min} / 1.73 \mathrm{~m}^{2}$ ) [normal (score=0)], between 50 to $75 \mathrm{ml} / \mathrm{min} / 1.73 \mathrm{~m}^{2}$ [ mild azotemia (score=1)], 25-49 ml $/ \mathrm{min} / 1.73 \mathrm{~m}^{2}$ [ moderate azotemia (score=2)], $<25 \mathrm{ml} / \mathrm{min} / 1.73 \mathrm{~m}^{2}$ [severe azotemia (score=3)].

\section{Pathological findings definitions:}

The criteria for enrollment were at least 5 glomeruli in light microscopic field, at least 1 segmentally sclerotic glomerulus.

The biopsy result included the number of glomeruli in biopsy specimens, the percentage of segmental glomerulosclerosis (SGS) (number of glomeruli with segmental sclerosis/all glomeruli $\times 100$ ) and percent of interstitial fibrosis/ tubular atrophy (IF/TA) of the cortical area. FSGS variant based on Columbia classification were extracted from the patients' files. The pathology slides were re-evaluated and reported in case of previously unclassified FSGS report.

Both SGS and IF/TA percentages were classified as 1-5\% [normal (score=0)], 6-25\% [mild (score=1)], 26-50\% [moderate (score=2)] and >50\% [severe (score=3)].

\section{Statistical Analyses and Development of Risk Prediction Model:}

Categorical data were presented as numbers and percentages, and continuous data as mean \pm SD. To compare the different clinical and laboratory characteristics of FSGS variants we used the Chi-2, one-way ANOVA and Post hoc Bonferoni tests, where appropriate. The correlation between SGS and IF/TA and laboratory findings were evaluated by Pearson test.

Continuous and categorical prognostic factors were analyzed by Cox regression model to estimate the risk of CKD/ESKD. Hazard Ratio (HR) with $95 \%$ confidence interval (Cl) was estimated by univariate and multivariate analyses. Possible interactions between significant variables were tested. A significant interaction was found between SGS and IF/TA percentages $(p=0.01)$ and also baseline SCr and IF/TA percentage $(p=0.05)$. As a result we calculated the product of two independent variables in model by using centered interaction.

According to the sum of the baseline scores of eGFR, SGS and IF/TA, the patients were classified into three groups of low risk (total score 0-3), medium risk (total score: 4-6) and high risk (total score: 7-9). Finally Kaplan-Meier and the log-rank methods were used to estimate renal survival in each group (Supplementary Tab 1 and 2).

In prognostic model, discrimination was evaluated using the C-statistic, which represents the area under the receiver operating characteristic curve (ROC) curve. Accuracy of the test was described as area under the ROC curve (AUC). An area of 1.0 reflects perfect discrimination (sensitivity and specificity both $100 \%$ ) and C-Statistic less than 0.5 is equivalent to random guessing. 
In Multivariate analyses each main independent variable was adjusted for other variables. In addition, due to significant interaction between IF/TA percentages and SGS and IF/TA and SCr at baseline, we added the main effect of their products in the model, as explained in methodology.

\section{Results}

Baseline characteristics: Baseline characteristics of 201 patients and histological variants are shown in table 1. The prevalence of FSGS variants by kidney biopsy was as follows: NOS (68\%), tip lesion (22\%), perihilar (6\%), collapsing (3\%), and cellular (1\%).

Clinical and laboratory findings at baseline: In 201 patients, the mean age was $38 \pm 15$ years and 119 were male (59\%). Eighty patients (40\%) were hypertensive at admission, however $70 \%$ of the patients with collapsing variant were hypertensive.

The mean SCr at baseline was $1.89 \pm 1.63 \mathrm{mg} / \mathrm{dl}$, eGFR at baseline was $65 \pm 38 \mathrm{ml} / \mathrm{min} / 1.73 \mathrm{~m}^{2}$ and 94 patients (47\%) had SCr>1.4 mg/dl. In collapsing lesion $88 \%$ of the patients had SCr $>1.4 \mathrm{mg} / \mathrm{dl}$, baseline $\mathrm{SCr}$ was significantly higher $(4.0 \pm 3.2 \mathrm{mg} / \mathrm{dl} ; \mathrm{P}<0.001)$, and eGFR was significantly lower $(27 \pm 19$ $\left.\mathrm{ml} / \mathrm{min} / 1.73 \mathrm{~m}^{2} ; \mathrm{P}<0.001\right)$ than others (table 1 ).

Mean serum albumin was $3.2 \pm 0.8 \mathrm{~g} / \mathrm{dl}$ and 24 -hour urine protein was $4.6 \pm 3.6 \mathrm{~g} / 24 \mathrm{~h}$ in all patients. Patients with tip lesion had the lowest serum albumin $(2.6 \pm 0.7 \mathrm{~g} / \mathrm{dl})$ and the highest amount of proteinuria $(4.0 \pm 4.6 \mathrm{~g} / 24 \mathrm{~h})$ at baseline.

Histological findings: The average number of glomeruli per one biopsy specimen was $19.8 \pm 9.8$. Most of the patients (72\%) had normal (0-5\%) to mild (6-25\%) SGS or IF/TA. SGS and IF/TA $>50 \%$ were only observed with collapsing and NOS variants.

Development of CKD, ESKD and non-response: In this cohort of FSGS patients with a mean follow up of $55 \pm 27$ months, $82(41 \%)$ patients developed CKD [46 patients (23\%)] or ESKD [36 patients (18\%)]. Ninety patients $(44 \%)$ did not respond to treatment and among them only 8 patients $(9 \%)$ did not develop CKD/ESKD. At the end of the study the patients had a mean SCr of $1.87 \pm 1.89 \mathrm{mg} / \mathrm{dl}$ and proteinuria of $0.76 \pm 0.96 \mathrm{~g} / 24 \mathrm{~h}$.

Risk Factors associated with renal outcome: Association between continuous and categorical independent variables and the risk of development of CKD/ESKD are shown in table 2.

Univariate analyses: In univariate analyses with continuous variables, higher $\mathrm{SCr}$ at baseline, higher percentage of glomeruli with SGS and higher IF/TA percentages were associated with increased risk of CDK/ESKD. Higher albuminuria at baseline was associated with slight increase of risk. In categorical variables hypertension was associated with increased risk of CKD/ESRD.

Multivariate analyses: In multivariate analysis, Scr at baseline and percentages of SGS and IF/TA were still significantly associated with development of CKD/ESKD. 
Histological variants and the risk of non-response and CKD/ESKD with primary FSGS:

Among 137 patients with NOS variant, 80 (58\%) were defined as non-responders and 71 cases (52\%) developed CKD/ESKD. In 11 patients with perihilar lesion, two (18\%) progressed to CKD/ESKD and did not respond to treatment. From 44 patients with tip lesion variant, only two (5\%) developed CKD/ESKD and both were defined as non-responders. The 2 cases with cellular variant achieved complete remission. None of the 7 cases with collapsing lesion (100\%) responded to treatment and all progressed to CKD/ESKD.

The risk of CKD/ESKD with histological variants of primary FSGS is given in table 3. In univariate analyses, with the NOS type defined as the reference group, the collapsing type was significantly associated with increased risk of developing CDK/ESKD (HR 5.24, 95\% Cl 2.38-11.36, P<0.001). However in multivariate model, when adjusted for pathological findings of SGS and IF/TA percentages, the risk of developing CDK/ESKD with collapsing lesion was not significant (HR 2.31, 95\% Cl 0.73-7.30, $\mathrm{P}=0.15$ ).

Risk category and renal survival: As mentioned before, the prognostic risk score was calculated according to the sum of the baseline scores of eGFR, SGS, IF/TA, and divided into three categories of low risk ( $\leq 3)$, medium risk (4-6 points), and high risk (7-9 points). 92(46\%) were classified as low risk, $83(41 \%)$ as medium risk, and $26(13 \%)$ as high risk. Renal survival by risk category is shown in Figure 1.The median renal survival was calculated at 6.7 years for all patients ( $95 \% \mathrm{Cl}, 6.1$ to 7.2 years), 8.1 years for low risk patients ( $95 \% \mathrm{Cl}, 7.7$ to 8.6$), 5.8$ years for medium-risk patients ( $95 \% \mathrm{Cl}, 5.1$ to 6.5 years), and 3.3 years for high-risk patients $(95 \% \mathrm{Cl}, 2.3$ to 4.2 years; $\mathrm{P}<0.001)$.

\section{Five years prediction of CKD/ESKD:}

The five years prediction of CKD/ESKD is given in figure 2. Receiver-operating characteristic curves (ROCcurve) with C-Statistic were used to estimate discrimination of the risk predictors.

Serum $\mathrm{Cr}$ at baseline and SGS and IF/TA percentages had C-Statistic of 0.83 (95\% Cl: 0.77-0.89), 0.82 (95\% Cl: $0.75-0.88)$ and $0.88,(95 \% \mathrm{Cl}: 0.83-0.94)$. Prediction of risk by using all three variables in prognostic risk score resulted in C-Statistic of 0.83 (95\% Cl: $0.77-0.90)$.

Baseline proteinuria as a predictor had C-Statistic less than $0.5(0.40,95 \% \mathrm{Cl}: 0.35-0.50)$ (figure 2$)$.

\section{Discussion}

FSGS is caused by podocyte loss and is mostly a progressive glomerular disease $(13,14)$. Renal outcome is very diverse and progression to advanced kidney failure is common $(15,16)$.

In the present study we assessed the predictors of CKD/ESKD in patients with primary FSGS. Forty- five percent of our patients did not respond to treatment and $41 \%$ developed CKD or ESKD. The link between remission and renal survival was very high and $90 \%$ of patients without remission developed CKD/ESKD. 
In a study by Chun et al. the 10 years' kidney survival rates of patients with FSGS was $92 \%$ in patients with remission compared to $33 \%$ in those without remission (2).

Despite including 6 patients younger than 18 years, the demographics of our patients are almost similar to other studies of adult FSGS, with a mean age of $38 \pm 15$ years in our study and an age at diagnose of $35-50$ years old in other studies $(11,15)$. The prevalence of idiopathic FSGS was somewhat more in men in our study similar to others $(14,17)$.

Most of the patients in this study had mild renal impairment at baseline (MDRD eGFR: $65 \pm 38$ $\left.\mathrm{ml} / \mathrm{min} / 1.73 \mathrm{~m}^{2}\right)$. Renal function at admission is very diverse in adult primary FSGS but it is mostly reported as mild to moderate renal dysfunction at admission $(18,19)$. Our finding like others showed higher renal function at admission was positively related to better renal outcome $(2,20)$.

In our study the most prevalent variant of FSGS was NOS (68\%), followed by tip lesion (22\%), perihilar $(6 \%)$, collapsing (3\%), and cellular types (1\%). Demographic features are different between various histologic subtypes of FSGS. Collapsing lesion has been reported with a higher prevalence in AfroAmerican patients and both tip lesion and collapsing variants have been more common in teenagers and adults compared to children $(11,12,21)$.

Most studies have reported NOS variant as the most prevalent type of FSGS which is similar to our study $(12,21)$. However it is suggested that longer duration of FSGS may be correlated with NOS variant, probably due to evolution of segmental sclerosing lesions from tip lesion over time, as a part of chronicity process (22).

Regarding renal outcome, collapsing lesion has been associated with worst renal outcome and more common in non-responders (23). In our study all of the seven patients with collapsing lesion developed CKD/ESRD, including the two patients who ended in dialysis.

Tip lesion variant has been associated with a significantly better kidney survival $(24,25)$. Among 44 patients with tip lesion, only two did not attain complete or partial remission and developed CKD. In the current study $58 \%$ of patients with NOS did not response to treatment and more than half $(52 \%)$ developed CKD/ESRD, which shows a worse prognosis in our NOS patients compared to other studies $(11,21,26)$. This may have been the center effect and due to referral of more complicated patients to our kidney hospital, the effect of later referral compared to the mentioned studies or a basically different epidemiology of the disease in our country, which needs further investigation. Of the eleven patients with perihilar lesion, two progressed to CKD $(18 \%)$ and were defined as non-responder. In the study by Thomas et al the patients with perihilar variant had good renal survival at 1 year (89\%) and 3 years (75\%) (21).

In our study the only two cases of cellular variant attained complete remission. In other studies cellular variant shows an intermediate prognosis between the two variants of collapsing and tip lesion (21). The 
low number of patients with cellular lesion in the present study does not let us to have any hard conclusions.

We showed that in the collapsing variant the risk of CKD/ESKD is significantly high in univariate analyses, however after adding IF/TA score in the adjusted multivariate model, this risk was no longer significant. So the high frequency of bad outcome in collapsing FSGS may be due to higher chronicity at diagnosis and this should be noticed and examined in other series.

There are some limitations in using Columbia classification as a predictor of clinical outcome. Different location of segmental lesions makes overlapping and heterogeneity between variants, which can lead to misdiagnosis by kidney biopsy (27-29). The accuracy of diagnosis highly depends on the number of glomeruli and sample size in biopsy $(30,31)$.

We showed that high IF/TA score at diagnosis strongly predicted the risk of CKD/ESKD in primary FSGS. The term interstitial fibrosis/tubular atrophy was first introduced in kidney allograft biopsy (32, 33). However today it is used as a predictor for progression of kidney failure, regardless of the type of the glomerular disease and interstitial fibrosis and tubular atrophy are well identified hallmarks for development of CKD (34-36)

The presence of global and segmental sclerosis in kidney biopsy indicates worse prognosis in kidney survival $(4,37)$. Global sclerosis is highly age-related and segmental sclerosis is the most important pathological finding in the typical FSGS (38). In this study we used the percentage of segmental sclerosis in the biopsy specimen as a predictor. Our finding showed that the higher number of glomeruli with segmental sclerosis on biopsy highly predicts the risk of CKD/ESKD.

In this study baseline proteinuria was not associated with increase in the risk of CDK/ESKD. This finding is contrary to the previous finding of proteinuria as an important risk factor for development of renal failure $(39,40)$. On the other hand in a very recent study on 466 patients with primary FSGS and proteinuria, followed for 1,4 and 8 months, by using a novel definition of remission, reduction of proteinuria was associated with better long-term renal outcomes(41). In a sub-analysis in our patients (not shown), greater decrease of proteinuria between baseline and last proteinuria was associated with decreased risk of CKD/ESRD [multivariate analysis (HR: 0.92, 95\% $\mathrm{Cl} 0.86-0.97$ )].

This study has several limitations. Since this is an observational study,

a cause-effect relationship cannot be established. The patients were treated by different physicians and treatment protocols or follow-up time may have had an influence on outcome. Time from early clinical manifestation and renal biopsy is diverse and this can lead to time bias. Laboratory data were reported by different laboratories which may have slightly affected the accuracy of the data. However the major strengths of this cohort study are scale adequate number of patients with primary FSGS, good number of events, very few missing data and examination of all kidney biopsy samples by one expert pathologist. 


\section{Conclusion}

Our study showed that regardless of the histologic variant, kidney function at admission and percentages of glomeruli with segmental sclerosis and of interstitial fibrosis and tubular atrophy at kidney biopsy have high sensitivity and specificity to predict the kidney outcome. This is a retrospective observational study and these findings need to be examined by further prospective studies, which should consider the effect of therapeutic regimens.

\section{Abbreviations}

FSGS: Focal segmental glomerulosclerosis

ESKD: End stage kidney disease

CKD: Chronic kidney disease

NOS: Not otherwise specified

SCr: Serum creatinine

eGFR: Estimated glomerular filtration rate

SGS: Segmental glomerulosclerosis

IF/TA: Interstitial fibrosis/ tubular atrophy

HR: Hazard Ratio

Cl: Confidence interval

ROC: Receiver operating characteristic curve

AUC: Area under curve

\section{Declarations}

Ethics approval and consent to participate:

Code of ethics:94/D/105/198

Ethics Committee of Iran University of Medical Sciences

Consent for publication:

Not applicable 
Availability of data and materials:

The datasets used and analyzed during the current study are available from

the corresponding author on reasonable request.

\section{Competing interests:}

The authors declare that they have no competing interests.

\section{Funding:}

Not applicable

\section{Authors' contributions:}

SO has designed study and wrote the manuscript. SO, MY, MA and HA analyzed data, and participated in writing the manuscript. HA performed statistical analyses. MY collected data. MA preformed renal pathological analysis. SO supervised the project. All of the authors read and approved the manuscript.

\section{Acknowledgement:}

We would like to thank all participating physicians, nurses, and other staff who have contributed to this study.

\section{Publisher's Note:}

Springer Nature remains neutral with regard to jurisdictional claims in published maps and institutional affiliations.

\section{Author details:}

${ }^{1}$ Department of Medicine- Nephrology Ward, Iran University of Medical Sciences, Hasheminejad Kidney Center, Tehran, Iran. ${ }^{2}$ Department of Pathology, Iran University of Medical Sciences- Hasheminejad Kidney center, Tehran, Iran

${ }^{3}$ Department of Nephrology, Skaraborg Hospital, Skövde, Sweden.

Disclosure Statement: The authors have no competing interests.

We can provide our data and material in a SPSS chart if needed. 


\section{References}

1. D'Agati VD, Fogo AB, Bruijn JA, Jennette JC. Pathologic classification of focal segmental glomerulosclerosis: a working proposal. Am J Kidney Dis. 2004;43(2):368-82.

2. Chun MJ, Korbet SM, Schwartz MM, Lewis EJ. Focal segmental glomerulosclerosis in nephrotic adults: presentation, prognosis, and response to therapy of the histologic variants. J Am Soc Nephrol. 2004;15(8):2169-77.

3. Koop K, Eikmans M, Baelde HJ, Kawachi H, De Heer E, Paul LC, et al. Expression of podocyteassociated molecules in acquired human kidney diseases. J Am Soc Nephrol. 2003;14(8):2063-71.

4. Cameron JS. The enigma of focal segmental glomerulosclerosis. Kidney Int Suppl. 1996;57:S119-31.

5. Gipson DS, Trachtman H, Kaskel FJ, Greene TH, Radeva MK, Gassman JJ, et al. Clinical trial of focal segmental glomerulosclerosis in children and young adults. Kidney Int. 2011;80(8):868-78.

6. Korbet SM. Treatment of primary focal segmental glomerulosclerosis. Kidney Int. 2002;62(6):230110.

7. Deegens JK, Assmann KJ, Steenbergen EJ, Hilbrands LB, Gerlag PG, Jansen JL, et al. Idiopathic focal segmental glomerulosclerosis: a favourable prognosis in untreated patients? Neth $\mathrm{J}$ Med. 2005;63(10):393-8.

8. Bagherzadegan H, Ossareh S, Asgari M. Clinocopathologic Correlation of renal biopsy findings- An update report from a trtiary center. Abstracts of European Dialysis \& Transplant Association (ERAEDTA) $55^{\text {th }}$. May 24-27, 2018, Copenhagen, Denmark

9. D'Agati V. The many masks of focal segmental glomerulosclerosis. Kidney Int. 1994;46(4):1223-41.

10. Collins AJ, Foley RN, Herzog C, Chavers BM, Gilbertson D, Ishani A, et al. Excerpts from the US Renal Data System 2009 Annual Data Report. Am J Kidney Dis. 2010;55(1 Suppl 1):S1-420, A6-7.

11. D'Agati VD, Alster JM, Jennette JC, Thomas DB, Pullman J, Savino DA, et al. Association of histologic variants in FSGS clinical trial with presenting features and outcomes. Clin J Am Soc Nephrol. 2013;8(3):399-406.

12. Stokes MB, Valeri AM, Markowitz GS, D'Agati VD. Cellular focal segmental glomerulosclerosis: Clinical and pathologic features. Kidney Int. 2006;70(10):1783-92.

13. Chen YM, Liapis H. Focal segmental glomerulosclerosis: molecular genetics and targeted therapies. BMC Nephrol. 2015;16:101.

14. Gbadegesin R, Lavin P, Foreman J, Winn M. Pathogenesis and therapy of focal segmental glomerulosclerosis: an update. Pediatr Nephrol. 2011;26(7):1001-15.

15. Korbet SM. Primary focal segmental glomerulosclerosis. J Am Soc Nephrol. 1998;9(7):1333-40.

16. Kitiyakara C, Eggers P, Kopp JB. Twenty-one-year trend in ESRD due to focal segmental glomerulosclerosis in the United States. Am J Kidney Dis. 2004;44(5):815-25.

17. Schwartz MM, Korbet SM. Primary focal segmental glomerulosclerosis: pathology, histological variants, and pathogenesis. Am J Kidney Dis. 1993;22(6):874-83. 
18. Swarnalatha G, Ram R, Ismal KM, Vali S, Sahay M, Dakshinamurty KV. Focal and segmental glomerulosclerosis: does prognosis vary with the variants? Saudi J Kidney Dis Transpl. 2015;26(1):173-81.

19. Chou YH, Lien YC, Hu FC, Lin WC, Kao CC, Lai CF, et al. Clinical outcomes and predictors for ESRD and mortality in primary GN. Clin J Am Soc Nephrol. 2012;7(9):1401-8.

20. Schwartz MM, Evans J, Bain R, Korbet SM. Focal segmental glomerulosclerosis: prognostic implications of the cellular lesion. J Am Soc Nephrol. 1999;10(9):1900-7.

21. Thomas DB, Franceschini N, Hogan SL, Ten Holder S, Jennette CE, Falk RJ, et al. Clinical and pathologic characteristics of focal segmental glomerulosclerosis pathologic variants. Kidney Int. 2006;69(5):920-6.

22. Howie AJ, Pankhurst T, Sarioglu S, Turhan N, Adu D. Evolution of nephrotic-associated focal segmental glomerulosclerosis and relation to the glomerular tip lesion. Kidney Int. 2005;67(3):9871001.

23. Laurin LP, Gasim AM, Derebail VK, McGregor JG, Kidd JM, Hogan SL, et al. Renal Survival in Patients with Collapsing Compared with Not Otherwise Specified FSGS. Clin J Am Soc Nephrol. 2016;11(10):1752-9.

24. Huppes W, Hene RJ, Kooiker CJ. The glomerular tip lesion: a distinct entity or not? J Pathol. 1988;154(2):187-90.

25. Kwon YE, Han SH, Kie JH, An SY, Kim YL, Park KS, et al. Clinical features and outcomes of focal segmental glomerulosclerosis pathologic variants in Korean adult patients. BMC Nephrol. 2014;15:52.

26. Deegens JK, Steenbergen EJ, Borm GF, Wetzels JF. Pathological variants of focal segmental glomerulosclerosis in an adult Dutch population--epidemiology and outcome. Nephrol Dial Transplant. 2008;23(1):186-92.

27. Taneda S, Honda K, Uchida K, Nitta K, Yumura W, Oda H, et al. Histological heterogeneity of glomerular segmental lesions in focal segmental glomerulosclerosis. Int Urol Nephrol. 2012;44(1):183-96.

28. Meehan SM, Chang A, Gibson IW, Kim L, Kambham N, Laszik Z. A study of interobserver reproducibility of morphologic lesions of focal segmental glomerulosclerosis. Virchows Arch. 2013;462(2):229-37.

29. Han MH, Kim YJ. Practical Application of Columbia Classification for Focal Segmental Glomerulosclerosis. Biomed Res Int. 2016;2016:9375753.

30. Corwin HL, Schwartz MM, Lewis EJ. The importance of sample size in the interpretation of the renal biopsy. Am J Nephrol. 1988;8(2):85-9.

31. Schachter AD. Computational simulation of renal biopsy accuracy in focal segmental glomerulosclerosis. Pediatr Nephrol. 2006;21(7):953-7.

32. Solez K, Axelsen RA, Benediktsson H, Burdick JF, Cohen AH, Colvin RB, et al. International standardization of criteria for the histologic diagnosis of renal allograft rejection: the Banff working 
classification of kidney transplant pathology. Kidney Int. 1993;44(2):411-22.

33. Remuzzi G, Bertani T. Pathophysiology of progressive nephropathies. N Engl J Med. 1998;339(20):1448-56.

34. Levey AS, Coresh J. Chronic kidney disease. Lancet. 2012;379(9811):165-80.

35. Zeisberg M, Neilson EG. Mechanisms of tubulointerstitial fibrosis. J Am Soc Nephrol. 2010;21(11):1819-34.

36. Bohle A, Muller GA, Wehrmann M, Mackensen-Haen S, Xiao JC. Pathogenesis of chronic renal failure in the primary glomerulopathies, renal vasculopathies, and chronic interstitial nephritides. Kidney Int Suppl. 1996;54:S2-9.

37. Elsherbiny HE, Alexander MP, Kremers WK, Park WD, Poggio ED, Prieto M, et al. Nephron hypertrophy and glomerulosclerosis and their association with kidney function and risk factors among living kidney donors. Clin J Am Soc Nephrol. 2014;9(11):1892-902.

38. Rule AD, Amer H, Cornell LD, Taler SJ, Cosio FG, Kremers WK, et al. The association between age and nephrosclerosis on renal biopsy among healthy adults. Ann Intern Med. 2010;152(9):561-7.

39. Chitalia VC, Wells JE, Robson RA, Searle M, Lynn KL. Predicting renal survival in primary focal glomerulosclerosis from the time of presentation. Kidney Int. 1999;56(6):2236-42.

40. Cameron JS, Turner DR, Ogg CS, Chantler C, Williams DG. The long-term prognosis of patients with focal segmental glomerulosclerosis. Clin Nephrol. 1978;10(6):213-8.

41. Troost JP, Trachtman H, Nachman PH, Kretzler M, Spino C, Komers R, et al. An Outcomes-Based Definition of Proteinuria Remission in Focal Segmental Glomerulosclerosis. Clin J Am Soc Nephrol. 2018;13(3):414-21.

\section{Figures}


Survival Functions

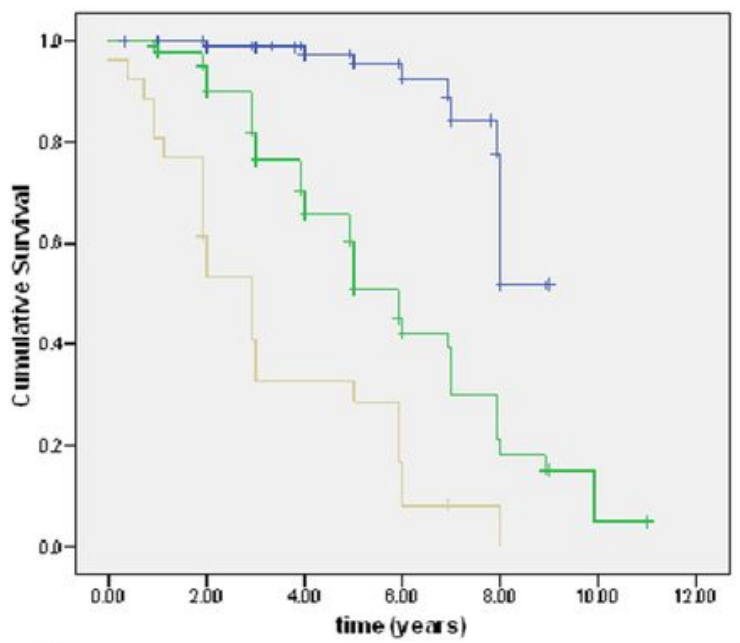

$\neg$ Medum Fisk Hgh Fosk

\begin{tabular}{|l|c|c|c|c|c|c|c|}
\hline & \multicolumn{9}{|c|}{ Numbers of patients at risk } \\
\hline Time(Years) & 0 & 2 & 4 & 6 & 8 & 10 & 12 \\
\hline Low risk & 92 & 77 & 65 & 30 & 8 & 1 & - \\
\hline Medium risk & 82 & 69 & 43 & 15 & 6 & 0 & - \\
\hline High Risk & 26 & 14 & 8 & 3 & 0 & 0 & - \\
\hline
\end{tabular}

\section{Figure 1}

Renal survival by risk category 


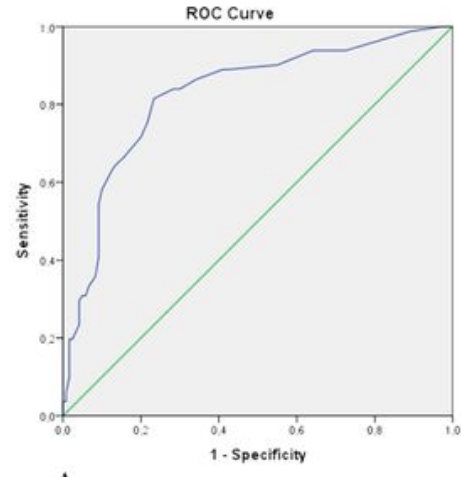

A Diagonal stgments ate pioduced by ties

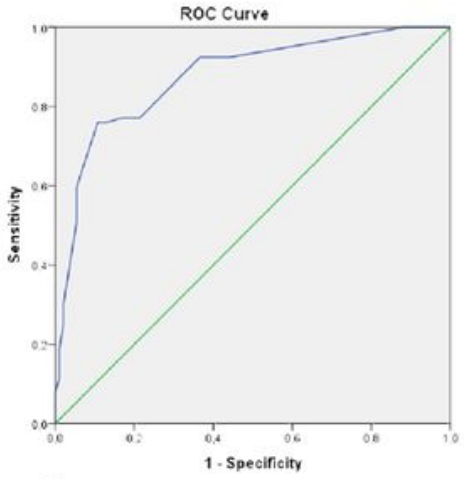

C

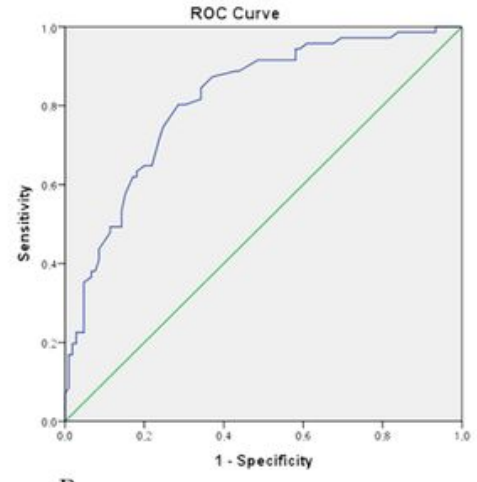

B

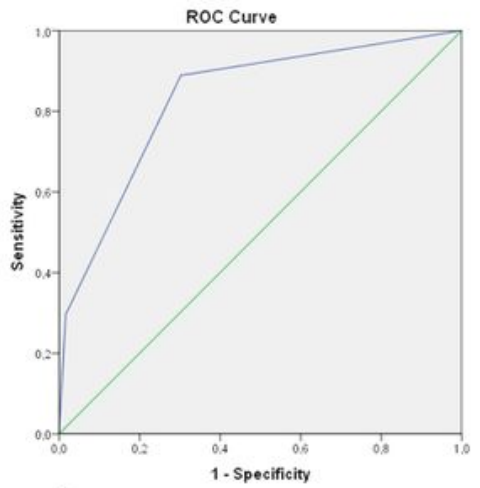

D Diagnal segments are preduced by tios

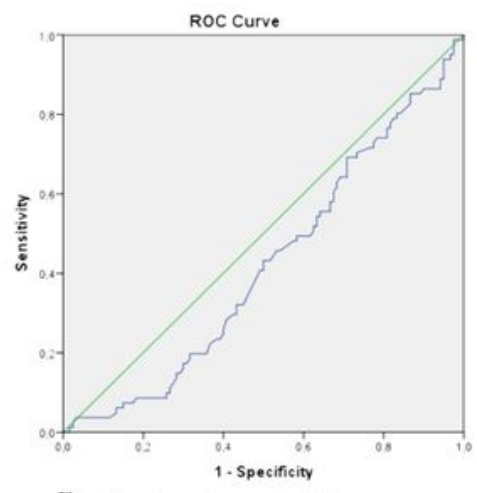

E Dagenal staments xe produced by tits

\section{Figure 2}

The five years prediction of CKD/ESKD

\section{Supplementary Files}

This is a list of supplementary files associated with this preprint. Click to download. 
- FSGSSupplementarytable1.pdf

- Table1.pdf

- Table2.pdf

- Table3.pdf

- FSGSSupplementarytable2.pdf 